\title{
FACTORIZATION STRUCTURES AND SUBCATEGORIES OF THE CATEGORY OF TOPOLOGICAL SPACES
}

\author{
RYOSUKE NAKAGAWA
}

(Received 2 July 1974)

Communicated by G. M. Kelly

Various investigations on $(E, M)$-categories are given by many authors. Recently, Herrlich (to appear) and Strecker (1972) gave interesting results on relations between factorizations $(E, M)$ on nice categories 5 and epireflective subcategories of $\mathfrak{S}$. Factorizations $(E, M)$ treated by them satisfy that $E \subset$ Epi $\mathfrak{S}$. In this paper we shall consider factorization structures $(E, M)$ such that $E \supset$ Epi $\mathfrak{V}$ in the category $\mathfrak{T}$ of all topological spaces and study relations between these structures and some subcategories of $\mathfrak{T}$. Our results are quite different from those in Herrlich (to appear) and Strecker (1972) and are rather related with the results in Herrlich (1969). Herrlich (1969) defined limit operators on $\mathfrak{T}$ and gave a one-to-one correspondence between suitable ${ }^{1}$ limit operators and bicoreflective subcategories of $\mathfrak{T}$. We shall consider two kinds of factorization structures on $\mathfrak{I}$ and show that the first ones correspond to bicoreflective subcategories which are closed under closed embeddings, the second ones correspond to bireflective subcategories which satisfy that the classes of all reflections are closed under closed embeddings (see Proposition 11) and these correspondences are one-to-one.

In $\$ 1$ we shall show a property of categories with factorization structures $(E, M)$ and define closure operations in these categories associated with $(E, M)$. We shall give a condition for an $(E, M)$-category to be embedded in the category $\mathfrak{I}_{-1}$ of all $T_{-1}$-spaces, where a $T_{-1}$-space is a generalized topological space with a closure operation which is monotone, inclusive and idempotent ( $\$ 2$ ). In $\$ 3$ stable and strong (weak) factorization structures on $\mathfrak{T}_{-1}$ will be defined. From these structures we shall construct bicoreflective (bireflective) subcategories of $\mathfrak{T}_{-1}$ satisfying the additional conditions stated above. In $\$ 4$ the inverse assignments

\footnotetext{
1 Answering the author's question, Herrlich showed that the condition on limit operators given by him (1969) is incomplete. In order to give the one-to-one correspondence we must consider limit operators satisfying the stability condition stated in this paper.
} 
are given. By considering additive factorization structures we can obtain the same correspondence in the category $\mathfrak{I}(\$ 5)$. Several examples will be given.

Throughout the paper let $\mathfrak{E}$ be a complete, cocomplete, locally small and colocally small category. We shall denote the classes of all objects, morphisms, isomorphisms, momomorphisms, epimorphisms and extremal monomorphisms in $\mathfrak{S}$ by $\mathrm{Ob} \mathfrak{C}$, Mor $\mathfrak{S}$, Iso $\mathfrak{S}$, Mono $\mathfrak{S}$, Epi $\mathfrak{S}$ and Ex mono $\mathfrak{S}$, respectively. Terminology not explained here is from Herrlich (1968) and Mitchell (1965).

1.

We shall recall a Galois correspondence (cf. Herrlich (to appear)). Let $A$ and $B$ be arbitrary classes and $\rho$ a subclass of $A \times B . \rho$ induces functions $L_{\rho}: P B \rightarrow P A$ and $R_{\mathrm{p}}: P A \rightarrow P B$ between the classes of all subclasses such that $L_{\rho}(Y)=\{a \in A \mid(a, y) \in \rho$ for all $y \in Y\}, \quad$ for $Y \subset B$ and $R_{\rho}(X)=$ $\{b \in B \mid(x, b) \in \rho$ for all $x \in X\}$, for $X \subset A$. A subclass $X$ of $A$ is called $L_{p}$-closed provided that $X=L_{\rho} Y$ for some $Y \subset B$ (equivalently: if and only if $\left.X=L_{\rho} R_{\rho} X\right)$. $R_{\rho}$-closed classes are also defined.

Let $\{$ be a complete, cocomplete, locally small and colocally small category and let $A=B=$ Mor $(\mathfrak{E}$. We shall consider a subclass $\delta$ of $A \times B$ such that $\delta=\{(e, f) \mid e \in A, f \in B$ and whenever $r$ and $s$ are morphisms with $f r=s e$ then there exists a morphism $d$ such that $d e=r$ and $f d=s\}$.

Let $E$ and $M$ be subclasses of Mor $\mathbb{E}$ which contain Iso $\mathbb{E}$. $(\mathcal{E}$ is called an $(E, M)$-category provided that $E$ and $M$ are subcategories of $\mathbb{S}$ and every morphism $f$ in $(5$ has a unique $(E, M)$-factorization $f=h g, g \in E, h \in M$. In this case $E$ is called a left factor of $(\mathcal{E}$ and $M$ a right factor of $\mathfrak{E}$. It is known that a complete and locally small category $(\mathfrak{S}$ is an (Epi $\mathfrak{S}$, Ex mono $(\mathfrak{S})$-category. In the category $\mathfrak{I}_{-1}$ (or $\mathfrak{T}$ ), let $E_{0}$ and $M_{0}$ be the classes of all continuous dense maps and all closed embeddings respectively. Then $\mathfrak{I}_{-1}($ or $\mathfrak{T})$ is an $\left(E_{0}, M_{0}\right)$-category.

Proposition 1. Suppose that $M$ is a subclass of Mor (5 such that $M \subset$ Ex mono 5 . Then the following conditions are equivalent.

(a) $M$ is $R_{\delta}$-closed.

(b) $M$ is a right factor of (5.

(c) (s is a $\left(L_{\delta} M, M\right)$-category.

(d) $M$ satisfies the following conditions.

(M1) $M$ is a subcategory of $E$ such that $M \supset$ Iso(5.

(M2) $M$ is closed under the formation of pullbacks.

(M3) $M$ is closed under the formation of intersections.

(e) $M$ satisfies the conditions (M1), (M2) and

(M4) $M$ is closed under the formation of products.

(f) $M$ satisfies the conditions $(M 1) \sim(M 4)$ and

(M5) if $g f \in M$ and $g \in$ Mono $(\mathcal{S}, f \in M$. 
The proof is easy and so omitted. $(E, M)$-categories are investigated by Kennison (1968), Herrlich (to appear), Strecker (1972) and others. For the case that $\boldsymbol{A}=\mathrm{Epi}(\mathfrak{S}$ and $\boldsymbol{B}=$ Mor $(5$ similar results are given by Herrlich (to appear) and Strecker (1972).

Remark. Let $M$ be an $R_{\delta}$-closed class of extremal monomorphisms in the categories $\mathfrak{T}_{-1}$ or $\mathfrak{T}$. Then if a coproduct $\oplus_{\lambda \in \Lambda} f_{\lambda}$ of morphisms $f_{\lambda}, \lambda \in \Lambda$ belongs to $L_{\delta} M$, each $f_{\lambda}$ belongs to $L_{\delta} M$. By using the fact that any morphism $f: X \rightarrow \oplus_{\lambda \in \Lambda} Y_{\lambda}$ is a coproduct $\oplus_{\lambda \in \Lambda} f_{\lambda}: \oplus_{\lambda \in \Lambda} X_{\lambda} \rightarrow \oplus_{\lambda \in \Lambda} Y_{\lambda}$ of morphisms $f_{\lambda}^{-}$, we can prove that $M$ is closed under coproducts.

Whenever $\mathfrak{S}$ is an $(E, M)$-category and $M \subset E x$ mono $\mathfrak{S}$ (equivalently, $E \supset$ Epi $(5),(E, M)$ will be called a factorization structure on $\sqrt{5}$. For two factorization structures $(E, M)$ and $\left(E^{\prime}, M^{\prime}\right)$ on $\mathfrak{C}$, we shall define an order < by $(E, M)<\left(E^{\prime}, M^{\prime}\right)$ if $M \subset M^{\prime}$. Let $\left(E_{i}, M_{i}\right)$ be a factorization structure on $\mathfrak{S}_{\text {, }}$, $i=1,2$. A functor $F$ from $\mathfrak{V}_{1}$ to $\mathfrak{V}_{2}$ is called compatible with the factorization structures provided that for a morphism $f$ in $\mathfrak{S}_{1}$ with an $\left(E_{1}, M_{1}\right)$-factorization $f=h g, F(f)$ has an $\left(E_{2}, M_{2}\right)$-factorization $F(f)=F(h) F(g)$.

Here we shall recall the definition of images and unions. Images and unions as subobjects of an object in $(5$ are already defined (for example, see Mitchell (1965)). We shall consider them as extremal subobjects of an object in $(\mathfrak{S}$. Their definitions can be accomplished by taking extremal monomorphisms instead of monomorphisms in the definitions stated in Mitchell (1965). For a morphism $f: X \rightarrow Y$ and an extremal monomorphism $i: A \rightarrow X$, let $f^{\prime}: A \rightarrow B$ and $j: B \rightarrow Y$ be an (Epi (5, Ex mono (5)-factorization of $f i$. Then we have that $j$ is an image of $i$ by $f$. For extremal monomorphisms $i_{\lambda}: A_{\lambda} \rightarrow X, \lambda \in \Lambda$ there exists a morphism $k: \bigoplus_{\lambda \in \Lambda} A_{\lambda} \rightarrow X$ such that $k j_{\lambda}=i_{\lambda}$, where $\bigoplus_{\lambda \in \Lambda} A_{\lambda}$ is a coproduct of $\left\{A_{\lambda}\right\}_{\lambda \in \Lambda}$ and $j_{\lambda}: A_{\lambda} \rightarrow \bigoplus_{\lambda \in \Lambda} A_{\lambda}$ is the injection. Let $g: \oplus_{\lambda \in \Lambda} A_{\lambda} \rightarrow A^{\prime}$ and $h: A^{\prime} \rightarrow A$ be (Epi (S, Ex mono (S)-factorization of $k$. Then we have that $h$ is a union of $\left\{i_{\lambda}\right\}_{\lambda \in A}$.

We shall denote an extremal subobject by $A \subset X$, an image of $A \subset X$ by $f: X \rightarrow Y$ by $f(A)$ and a union of $\left\{A_{\lambda}\right\}_{\lambda \in \Lambda}$ by $\bigcup_{\lambda \in \Lambda} A$.

Now let $(E, M)$ be a factorization structure on $(5$. For an extremal monomorphism $i: A \rightarrow X, i: A K X$, let $j ; A \rightarrow A^{\prime}$ and $k: A^{\prime} \rightarrow X$ be an $(E, M)$ factorization of $i$. Define a closure $\mathrm{cl}_{X}(A)$ of an extremal subobject $A \subset X$ by $\mathrm{cl}_{x}(A)=A^{\prime}$. cl will be called a closure operation in $(5$ associated with a factorization structure $(E, M)$. From Proposition 1 we have the following.

Proposition 2. For any morphism $f: X \rightarrow Y$ and an extremal subobject $A \subset X, f\left(\operatorname{cl}_{X}(A)\right) \subset \mathrm{cl}_{Y}(f(A))$.

Proposition 3.

(1) $\mathrm{cl}_{X}(X)=X$. 
(2) If $A \subset B \subset X, \operatorname{cl}_{x}(A) \subset \mathrm{cl}_{x}(B)$.

(3) $A \subset \mathrm{cl}_{x}(A)$.

(4) $\operatorname{cl}_{X}(A)=\operatorname{cl}_{X}\left(\operatorname{cl}_{X}(A)\right)$.

A factorization structure $(E, M)$ will be called additive if it satisfies the following condition.

(A) If $i: A \rightarrow X$ and $j: B \rightarrow X$ belongs to $M$, their union $k: A \cup B \rightarrow X$ belongs to $M$.

Let $i: A \rightarrow X$ and $j: B \rightarrow X$ be any extremal monomorphisms and $i_{0}: A \rightarrow A_{1}, i_{1}: A_{1} \rightarrow X$ and $j_{0}: B \rightarrow B_{1}, j_{1}: B_{1} \rightarrow X$ be $(E, M)$-factorizations of $i$ and $j$ respectively. $i_{0}, j_{0}$ and $i_{1}, j_{1}$ determine morphisms $k_{0}: A \cup B \rightarrow A_{1} \cup B_{1}$ and $k_{1}: A_{1} \cup B_{1} \rightarrow X$ respectively. It can be shown that the following condition is equivalent to (A).

$\left(\mathrm{A}^{\prime}\right)$ For any extremal monomorphisms $i: A \rightarrow X$ and $j: B \rightarrow X,\left(k_{0}, k_{1}\right)$ is an $(E, M)$-factorization of their union $k: A \cup B \rightarrow X$.

Proposition 4. If $(E, M)$ is an additive factorization structure, then for any $A$ and $B \subset X, \operatorname{cl}_{X}(A \cup B)=\operatorname{cl}_{X} A \cup \mathrm{cl}_{X} B$.

2.

An object $X$ in $\left(\mathcal{S}\right.$ is called connected if for any family $\left\{Y_{\lambda}\right\}_{\lambda \in \Lambda}$ of objects and any morphism $f: X \rightarrow \oplus_{\lambda \in \Lambda} Y_{\lambda}$, there exists a unique index $\lambda$ and a morphism $f_{\lambda}: X \rightarrow Y_{\lambda}$ such that $X \in \mathrm{Ob}\left(\mathcal{S}\right.$, let $H^{X}$ be the covariant morphism functor from $\sqrt{5}$ to the category $\subseteq$ of all sets. Then it is obvious that $H^{X}$ is a coproduct preserving functor if and only if $X$ is connected. An object $X$ in $\mathfrak{F}$ is called simple if $[X, X]$ has precisely one element, where $[A, B]$ means a set of morphisms from an object $A$ to an object $B$ in $\mathfrak{S}$.

Proposition 5. Suppose that 5 has a connected and projective generator $P$. Then $P$ is simple if and only if for any $X \in \mathrm{Ob} \mathfrak{C}$ and any non empty subset $S$ of $[P, X]$ there exists a unique extremal monomorphism $f_{s}: X_{s} \rightarrow X$ such that the induced map $H^{P}\left(f_{s}\right):\left[P, X_{s}\right] \rightarrow[P, X]$ coincides with the inclusion map $i: S \rightarrow[P, X]$.

Proof. Let $X_{s}^{\prime}=\bigoplus_{\sigma \in S} P$ and $f_{s}^{\prime}: X_{s}^{\prime} \rightarrow X$ be a morphism such that $f_{s}^{\prime} j_{\sigma=\sigma}$, where $j_{\sigma}:: P \rightarrow \oplus_{\sigma \in S} P$ is the injection. Let $g_{s}: X_{s}^{\prime} \rightarrow X_{s}$ and $f_{s}: X_{s} \rightarrow X$ be an (Epi $\left(\mathfrak{S}\right.$, Ex mono (5)-factorization of $f_{s}^{\prime}$. If $P$ is a simple, connected and projective generator, we have that $f_{s}$ is a desired morphism. Suppose that $\tilde{f}_{s}: \tilde{X}_{s} \rightarrow X$ is another morphism having the desired properties. Then there exists a morphism $h_{s}: X_{s}^{\prime} \rightarrow \tilde{X}_{s}$ such that $\tilde{f}_{s} h_{s}=f_{s}^{\prime}$ and it is shown that $h_{s}$ is an epimorphism. Thus we have an isomorphism $i: X_{s} \rightarrow \tilde{X}_{s}$ such that $i g_{s}=h_{s}$ and $\tilde{f}_{s} i=f_{s}$ and this means the uniqueness of $f_{s}$. The 'if' part can be obtained by considering the extremal subobject $X_{s}$ of $P$ for $S=\left\{1_{p}\right\} \subset[P, P]$. 
COROLlary. (1) $X_{[P, X]}=X$.

(2) If $S \subset T \subset[P, X], X_{S} \subset X_{T} \subset X$.

(3) If $S, T \subset[P, X], X_{S \cup T}=X_{S} \cup X_{T}$.

(4) If $k: X \rightarrow Y, k\left(X_{s}\right)=Y_{H^{P}(k)(s)}$.

Let $(5$ have a factorization structure $(E, M)$ and a simple, connected and projective generator $P$. For an object $X$ and non empty subset $S \subset[P, X]$, let $\operatorname{cl}(S)=\left[P, \operatorname{cl}_{X}\left(X_{S}\right)\right]$ and for the empty subset $\phi \subset[P, X]$, let $\operatorname{cl}(\phi)=\phi$, where $\operatorname{cl}_{X}$ is a closure operation associated with $(E . M)$. Then this operation defines a $T_{-1}$-topology on $[P, X]$. For $k: X \rightarrow Y \in$ Mor $\mathcal{E}, H^{P}(k):[P, X] \rightarrow[P, Y]$ becomes a continuous map between $T_{-1}$-spaces defined as above. When $(E, M)$ is additive, $[P, X]$ becomes a topological space.

THEOREM 1. Let $(5$ have a factorization structure $(E, M)$ and a simple, connected and projective generator $P$. Then the morphism functor $H^{P}$ induces an embedding functor $H^{P}$ from $\left(5\right.$ to $\mathfrak{I}_{-1}$ which is compatible with the factorization structures $(E, M)$ and $\left(E_{0}, M_{0}\right)$. If $(E, M)$ is additive, $H^{P}$ can be considered as an embedding functor from $\mathfrak{S}$ to $\mathfrak{I}$ compatible with the factorization structures $(E, M)$ and $\left(E_{0}, M\right)$, where $E_{0}$ and $M_{0}$ are the classes of all continuous dense maps and all closed embeddings in $\mathfrak{I}_{-1}$ or $\mathfrak{I}$ respectively.

Proof. We shall show the compatibility of $H^{P}:\left(\mathfrak{S} \rightarrow \mathfrak{T}_{-1}\right.$. It is obvious that if $k \in M$ then $H^{P}(k) \in M_{0}$. Suppose that $k: X \rightarrow Y \in E$ and let $H^{P}(k)=$ $\bar{h} \bar{g}, \bar{g}:[P, X] \rightarrow S, \bar{h}: S \rightarrow[P, Y]$ be an $\left(E_{0}, M_{0}\right)$-factorization in $\mathfrak{I}_{-1}$. Then there exists an extremal monomorphism $h: Y_{S} \rightarrow Y$ such that $H^{P}(h)=\bar{h}$. Let $X^{\prime}=$ $\oplus_{\sigma \in[P, X]} P$. Then we can define an epimorphism $l: X^{\prime} \rightarrow X$ and a morphism $m: X^{\prime} \rightarrow Y_{s}$ such that $h m=k l$. There exists a morphism $g: X \rightarrow Y_{s}$ such that $k=h g$ and $H^{P}(g)=\bar{g}$. $\bar{h} \in M_{0}$ means $h \in M$ and since $k \in E, h$ is an isomorphism. Hence so is $\bar{h}$ and we have that $H^{P}(k) \in E_{0}$.

By the above proof we have the following. Let $k: X \rightarrow Y \in$ Mor $\mathbb{S}$ and $H^{P}(k)=\bar{h} \bar{g}$ be an $\left(E_{0}, M_{0}\right)$-factorization in $\mathfrak{T}_{-1}$. Then we have morphisms $g$ and $h$ such that $H^{P}(g)=\bar{g}, H^{P}(h)=\bar{h}$ and $k=h g$ is an $(E, M)$-factorization in (5.

\section{3.}

In this section we shall be concerned with the category $\mathfrak{I}_{-1}$. Let $P$ be a one point space, $E_{0}$ the class of all continuous dense maps, $M_{0}$ the class of all closed embeddings in $\mathfrak{I}_{-1}, E_{1}=$ Epi $\mathfrak{I}_{-1}$ and $M_{1}=$ Ex mono $\mathfrak{I}_{-1}$. Then $P$ is a simple, connected and projective generator in $\mathfrak{I}_{-1}$ and $\left(E_{0}, M_{0}\right)$ and $\left(E_{1}, M_{1}\right)$ are factorization structures on $\mathfrak{I}_{-1}$. Throughout the rest of the paper factorization structures $(E, M)$ on $\mathfrak{I}_{-1}$ or $\mathfrak{I}$ are assumed to satisfy that for any object $X$ a morphism $\phi \rightarrow X$ belongs to $M$. Let $(E, M)$ be a factorization structure on $\mathfrak{I}_{-1}$. By using this structure we obtain an embedding functor $H^{P}: \mathfrak{I}_{-1} \rightarrow \mathfrak{I}_{-1}$ which is compatible with $(E, M)$ and $\left(E_{0}, M_{0}\right)$. We shall denote $H^{P}$ by $F_{(E, M)}$ and define 
full subcategories $\mathfrak{I}_{-1}(E, M)$ and $\mathfrak{I}_{-1}^{\prime}(E, M)$ of $\mathfrak{T}_{-1}$ as follows. $O b \mathfrak{I}_{-1}(E, M)$ consists of all $T_{-1}$-spaces $X$ such that $F_{(E, M)} X=X$; this is equivalent to the condition that any subspace $A$ of $X$ is closed in $X$ if and only if the inclusion map $i: A \rightarrow X$ belongs to $M$. Ob $\mathfrak{I}_{-1}^{\prime}(E, M)$ consists of all $T_{-1}$-spaces $X$ such that $X=F_{(E, M)} Y$ for some $Y \in \mathrm{Ob} \mathfrak{I}_{-1}$. It is obvious that $\mathfrak{I}_{-1}(E, M) \subset \mathfrak{I}_{-1}^{\prime}(E, M)$ and there exists an example such that $\mathfrak{I}_{-1}(E, M) \neq \mathfrak{I}_{-1}^{\prime}(E, M)^{2}$.

Proposition 6. If $(E, M)>\left(E_{0}, M_{0}\right), \mathfrak{I}_{-1}(E, M)$ is a bicoreflective subcategory of $\mathfrak{I}_{-1}$ which is closed under closed embeddings.

Proof. It can be shown by the same way as is given in Herrlich (1969) that $\mathfrak{T}_{-1}(E, M)$ is bicoreflective in $\mathfrak{T}_{-1}$. Let $f: X \rightarrow Y$ be a closed embedding, $Y \in \mathrm{Ob} \mathfrak{I}_{-1}(E, M)$ and $i: A \rightarrow X$ belong to $M$. Then $f i$ belongs to $M$. Since $Y \in \mathrm{Ob} \mathfrak{I}_{-1}(E, M), f i$ is a closed embedding and so is $i$. Thus we have that $X \in \mathrm{Ob} \mathfrak{I}_{-1}(E, M)$.

Proposition 7. The following conditions for a factorization structure $(E, M)$ with $(E, M)>\left(E_{0}, M_{0}\right)$ are equivalent.

(a) $F_{(E, M)} F_{(E, M)}=F_{(E, M)}$.

(b) $\mathfrak{I}_{-1}^{\prime}(E, M)=\mathfrak{I}_{-1}(E, M)$.

(c) A bimorphism $f: X \rightarrow Y$ satisfying the condition $\left(m^{0}\right)$ satisfies the condition $(m)$.

$\left(m^{0}\right)$ An extremal monomorphism $i: A \rightarrow X$ belongs to $M_{0}$ if and only if the image $j: f(A) \rightarrow Y$ of $i$ by $f$ belongs to $M$.

(m) An extremal monomorphism $i: A \rightarrow X$ belongs to $M$ if and only if the image $j: f(A) \rightarrow Y$ of $i$ by $f$ belongs to $M$.

(d) A bimorphism $f: X \rightarrow Y$ satisfying the condition $\left(m^{0^{\prime}}\right)$ below satisfies the above condition $(m)$.

$\left(m^{o^{\prime}}\right)$ If an extremal monomorphism $i: A \rightarrow X$ belongs to $M_{0}$, the image $j: f(A) \rightarrow Y$ of $i$ by $f$ belongs to $M$.

It is noted that the condition $\left(m^{0}\right)$ on a bimorphism $f: X \rightarrow Y$ is equivalent to each of the following.

( $\left.m^{0} 1\right)$ If $i_{0}: A \rightarrow A_{1}, i_{1}: A_{1} \rightarrow X$ form the $\left(E_{0}, M_{0}\right)$-factorization of an extremal monomorphism $i: A \rightarrow X$, their images $j_{0}: f(A) \rightarrow f\left(A_{1}\right), j_{1}:\left(A_{1}\right) \rightarrow Y$ form the $(E, M)$-factorization of the image $j: f(A) \rightarrow Y$ of $i$ by $f$.

$\left(m^{0} 2\right)$ If $j_{0}: B \rightarrow B_{1}, j_{1}: B_{1} \rightarrow Y$ form the $(E, M)$-factorization of an extremal monomorphism $j: B \rightarrow Y$, their inverse images $i_{0}: f^{-1}(B) \rightarrow f^{-1}\left(B_{1}\right)$,

2 In his letter to the author, Herrlich stated that there exist several examples of the above statement and kindly showed the following example due to S. H. Kamnitzer: let $\boldsymbol{M}^{\prime}$ be a subclass of Ex mono $\mathfrak{I}_{-1}$ consisting of all closed embeddings and all open embeddings. Then there exists a smallest additive factorization structure $(E, M)$ such that $(E, M)>\left(L_{8} M^{\prime}, R_{8} L_{8} M^{\prime}\right)$. For the reals $R$ with the upper topology we have $F_{(E, M)} R \notin \mathfrak{T}_{-1}(E, M)$. 
$i: f^{-1}\left(B_{1}\right) \rightarrow X$ form the $\left(E_{0}, M_{0}\right)$-factorization of the inverse image $i$ of $i$.

Similar facts hold for the above condition $(m)$ and the condition $\left(m_{0}\right)$ in Proposition 10 below.

Proof. The equivalence of (a), (b) and (c) and that (d) implies (c) are obtained immediately. Suppose that (a) is satisfied and that $f: X \rightarrow Y$ is a bimorphism satisfying $\left(\mathrm{m}^{\circ}\right)$. Then by Proposition 8 below we obtain the $\mathfrak{I}_{-1}(E, M)$-coreflection $a_{Y}: F_{(E, M)} Y \rightarrow Y$ of $Y$. Let $g: F_{(E, M)} Y \rightarrow X$ be a map between underlying sets such that $f g=a_{Y}$. For $i: A \rightarrow X \in M_{0}$, the image $j: f(A) \rightarrow Y \in M$. From the definition of $F_{(E, M)} Y, k: a_{Y}^{-1} f(A) \rightarrow F_{(E, M)} Y \in M_{0}$. This implies that $g$ is continuous. Hence for $i: A \rightarrow X \in M$, the inverse image $k: \dot{g}^{-1}(A) \rightarrow F_{(E, M)} Y \in M$. Since $F_{(E, M)} Y \in O b \mathfrak{T}_{-1}(E, M)$, we have $k \in M_{0}$ and that the image $j: a_{Y} g^{-1}(A)=f A \rightarrow Y \in M$. Therefore we have that $f$ satisfies $(m)$.

A factorization structure $(E, M)$ such that $(E, M)>\left(E_{0}, M_{0}\right)$ will be called strong and moreover if one of the conditions in Proposition 7 is satisfied will be called stable.

Proposition 8. If $(E, M)$ is a strong and stable factorization structure on $\mathfrak{I}_{-1}, F_{(E, M)} X$ is a $\mathfrak{T}_{-1}(E, M)$-coreflection of $X \in \mathrm{Ob} \mathfrak{T}_{-1}$.

Proof. That $(E, M)>\left(E_{0}, M_{0}\right)$ means that the identity map $a_{X}: F_{(E . M)} X \rightarrow X$ between underlying sets is continuous. For $Y \in \mathrm{Ob}_{-1}(E, M)$ and $g: Y \rightarrow X \in$ Mor $\mathfrak{I}_{-1}$, there is a map $h: Y \rightarrow F_{(E, M)} X$ between underlying sets such that $a_{X} h=g$. We can show that $h$ is continuous. The assumption that $(E, M)$ is stable means that $F_{(E, M)} X \in \mathrm{Ob}_{-1}(E, M)$. Thus we have that $a_{X}$ is a coreflection.

When a factorization structure $(E, M)$ satisfies $(E, M)<\left(E_{0}, M_{0}\right)$, we have the following.

Proposition 9. If $(E, M)<\left(E_{0}, M_{0}\right), \mathfrak{I}_{-1}(E, M)$ is an epireflective subcategory of $\mathfrak{I}_{-1}$ which is closed under coproducts.

Proof. Let $X_{\lambda} \in \mathrm{Ob} \mathfrak{T}_{-1}(E, M), \lambda \in \Lambda, X=\times_{\lambda \in \Lambda} X_{\lambda}$ and $p_{\lambda}: X \rightarrow X_{\lambda}$ be the projection. Then $F_{(E, M)}\left(p_{\lambda}\right): F_{(E, M)}(X) \rightarrow F_{(E, M)}\left(X_{\lambda}\right)=X_{\lambda} \quad$ induces $p: F_{(E, M)}(X) \rightarrow X$. Since the identity map $b_{X}: X \rightarrow F_{(E, M)}(X)$ between underlying sets is continuous, we have that $F_{(E, M)}(X)=X$. It is easy to show that $\mathfrak{T}_{-1}(E, M)$ is closed under extremal monomorphisms. That $\mathfrak{T}_{-1}(E, M)$ is closed under coproducts follows from the remark of Proposition 1.

Proposition 10. The following conditions for a factorization structure $(E, M)$ with $(E, M)<\left(E_{0}, M_{0}\right)$ are equivalent.

(a) $F_{(E, M)} F_{(E, M)}=F_{(E, M)}$.

(b) $\mathfrak{I}_{-1}^{\prime}(E, M)=\mathfrak{I}_{-1}(E, M)$. 
(c) A bimorphism $f: X \rightarrow Y$ satisfying the condition $\left(m_{0}\right)$ below satisfies the condition $(m)$ in Proposition 7.

$\left(m_{0}\right)$ An extremal monomorphism $i: A \rightarrow X$ belongs to $M$ if and only if the image $j: f(A) \rightarrow Y$ belongs to $M_{0}$.

(d) A bimorphism $f: X \rightarrow Y$ satisfying the condition ( $\left.m_{0}^{\prime}\right)$ below satisfies the condition $(m)$ in Proposition 7.

( $\left.m_{0}^{\prime}\right)$ If an extremal monomorphism $i: A \rightarrow X$ belongs to $M$, the image $j: f(A) \rightarrow Y$ belongs to $M_{0}$.

A factorization structure $(E, M)$ such that $(E, M)<\left(E_{0}, M_{0}\right)$ will be called weak and moreover if one of the conditions of Proposition 10 is satisfied it will be called stable.

Proposition 11. If $(E, M)$ is weak and stable factorization structure on $\mathfrak{I}_{-1}, \mathfrak{I}_{-1}(E, M)$ is a bireflective subcategory of $\mathfrak{T}_{-1}$ and $F_{(E, M)} X$ is a $\mathfrak{T}_{-1}(E, M)$ reflection of $X \in \mathrm{Ob} \mathfrak{I}_{-1}$. Moreover the class of all $\mathfrak{T}_{-1}(E, M)$-reflections is closed under closed embeddings; that is, for any reflection $b_{X}: X \rightarrow F_{(E, M)} X$ and any closed embedding $f: B \rightarrow F_{(E, M)} X, b^{\prime}=b_{X} \mid A: A \rightarrow B$ coincides with the reflection $b_{A}$, where $A=b_{x}^{-1}(B)$.

The proofs of Propositions 10 and 11 are similar to those of Propositions 7 and 8.

4.

In this section we shall consider the converse of Propositions 8 and 11.

Let $\mathfrak{A}$ be a bicoreflective subcategory of $\mathfrak{T}_{-1}$ which is closed under closed embeddings, $a: \mathfrak{I}_{-1} \rightarrow \mathfrak{A}$ the coreflector and $a_{X}: a X \rightarrow X$ the coreflection of $X \in O b \mathfrak{I}_{-1}$. Define a class $M(\mathfrak{H})$ of morphisms in $\mathfrak{I}_{-1}$ by $M(\mathfrak{A})=$ $\left\{f: A \rightarrow X \in\right.$ Ex mono $\mathfrak{I}_{-1} \mid$ the inverse image $f^{\prime}: a_{X}^{-1}(A) \rightarrow a X$ of $f$ by $a_{X}$ is a closed embedding\}. We can show that an extremal monomorphism $f$ belongs to $M(\mathscr{I})$ if and only if $a f: a A \rightarrow a X$ is a closed embedding and that in this case $f^{\prime}$ coincides with af.

Proposition 12. ( $\left.L_{\delta} M(\mathfrak{H}), M(\mathfrak{X})\right)$ is a strong and stable factorization structure on $\mathfrak{T}_{-1}$.

Proof. It easily verified that $M(\mathfrak{A}) \supset M_{0}$ and $M(\mathfrak{A})$ satisfies the conditions (M1), (M2) and (M3) in Proposition 1 (d). It remains to show the stability. Let $f: X \rightarrow Y$ be a bimorphism satisfying the condition that if $i: A \rightarrow X \in M_{0}$, the image $j: f(A) \rightarrow Y$ of $i$ by $f$ belongs to $M(\mathfrak{H})$. We have a map $l: a Y \rightarrow X$ between underlying sets with $f l=a_{Y}$. Let $i: A \rightarrow X \in M_{0}$. Then the image $j: f(A) \rightarrow Y \in M(\mathfrak{A})$. By the definition of $M(\mathfrak{U}), j \mid a_{Y}^{-1} f(A): a_{Y}^{-1} f(A) \rightarrow a Y$ is a closed embedding. This implies that $l$ is continuous. Hence we can identify $l: a Y \rightarrow X$ with $a_{X}: a X \rightarrow X$. Let $i: A \rightarrow X \in M(\mathfrak{H})$ and $j: f(A) \rightarrow Y$ be its 
image. Then $a i \in M_{0}$ and hence we have $a j \in M_{0}$ by the above identification. This implies $j \in M(\mathfrak{A})$ and that $\left(L_{\delta} M(\mathscr{U}), M(\mathfrak{A})\right)$ is stable.

From Propositions 8 and 12, we have the following.

THEOREM 2. Suppose that $\mathfrak{A}$ is a bicoreflective subcategory of $\mathfrak{I}_{-1}$ which is closed under closed embeddings. Then $\mathfrak{I}_{-1}\left(L_{\delta} M(\mathfrak{A}), M(\mathfrak{X})\right)=\mathfrak{A}$. Suppose that $(E, M)$ is a strong and stable factorization structure on $\mathfrak{I}_{-1}$. Then $M\left(\mathfrak{I}_{-1}(E, M)\right)=$ $M$. In other words, there is a one-to-one correspondence between strong and stable factorization structures on $\mathfrak{I}_{-1}$ and bicoreflective subcategories of $\mathfrak{I}_{-1}$ which are closed under closed embeddings.

Next, suppose that $\mathfrak{B}$ is a bireflective subcategory of $\mathfrak{T}_{-}$, which satisfies that the class of all reflections is closed under closed embeddings (see Proposition 11). Let $b: \mathfrak{I}_{-1} \rightarrow \mathfrak{B}$ be the reflector and $b_{X}: X \rightarrow b X$ the reflection of $X \in \mathrm{Ob} \mathfrak{I}_{-1}$. We shall define a class $N(\mathfrak{B})$ of morphisms in $\mathfrak{T}_{-1}$ by $N(\mathfrak{B})=$ $\left\{f: A \rightarrow X \in\right.$ Ex mono $\mathfrak{I}_{-1} \mid$ the image $f^{\prime}: b_{X}(A) \rightarrow b X$ of $f$ by $b_{X}$ is a closed embedding\}. It can be shown that an extremal monomorphism $f: A \rightarrow X$ belongs to $N(\mathfrak{B})$ if and only if there exist morphisms $h: X \rightarrow Y, h^{\prime}: A \rightarrow B$ and $g: B \rightarrow Y$ such that $f, h^{\prime}, g, h$ form a pullback diagram, $Y \in O b \mathscr{B}$ and $g$ is a closed embedding. Then we can obtain the following.

Proposition 13. ( $\left.L_{\delta} N(\mathfrak{B}), N(\mathfrak{B})\right)$ is a weak and stable factorization structure on $\mathfrak{I}_{-1}$.

THEOREM 3. Suppose that $\mathfrak{B}$ is a bireflective subcategory of $\mathfrak{I}_{-1}$ which satisfies that the class of all reflections is closed under closed embeddings. Then $\mathfrak{I}_{-1}\left(L_{\delta} N(\mathfrak{B}), N(\mathfrak{B})\right)=\mathfrak{B}$. Suppose that $(E, M)$ is a weak and stable factorization structure on $\mathfrak{I}_{-1}$. Then $N\left(\mathfrak{I}_{-1}(E, M)\right)=M$. In other words, there exists a one-toone correspondence between weak and stable factorization structures on $\mathfrak{T}_{-1}$ and bireflective subcategories which satisfy that the classes of all reflections are closed under closed embeddings.

5.

In this section we shall consider the category $\mathfrak{T}$ of all topological spaces and continuous maps. Suppose that a factorization structure $(E, M)$ on $\mathfrak{T}$ is additive. Then we have an embedding functor $H^{P}: \mathfrak{I} \rightarrow \mathfrak{I}$ and we can define full subcategories $\mathfrak{T}(E, M)$ and $\mathfrak{T}^{\prime}(E, M)$ of $\mathfrak{T}$ and the stability of $(E, M)$ by the same way as in $\$ 3$. For a bicoreflective subcategory $\mathfrak{U}$ and a bireflective subcategory $\mathfrak{B}$ of $\mathfrak{T}$, define classes $M(\mathfrak{A})$ and $N(\mathfrak{B})$ by the same way as in $\S 4$. Then we can obtain that Theorems 2 and 3 hold in the category $\mathfrak{T}$ as follows.

THEOREM 4. There exists a one-to-one correspondence between additive, strong and stable factorization structures on $\mathfrak{I}$ and bicoreflective subcategories of $\mathfrak{T}$ which are closed under closed embeddings and there exists a one-to-one corres- 
pondence between additive, weak and stable factorization structures on $\mathfrak{T}$ and bireflective subcategories of $\mathfrak{I}$ which satisfy that the classes of all reflections are closed under closed embeddings.

REMARK. For an additive and strong factorization structure $(E, M)$ on $\mathfrak{I}$ we defined an associated closure operation $\mathrm{cl}$ in $\$ 1$. This is an idempotent limit operator defined in Herrlich (1969) and $\mathfrak{I}(E, M)$ is the category he denotes by $\mathfrak{I}(\mathrm{cl})$. Hence the first part of Theorem 4 is considered as a correct specialization of the one-to-one correspondence given by Herrlich (1969) (cf. Foot-note 1)). Conversely, for a limit operator $l$ we can define a class $M(l)$ of morphisms in $\mathfrak{I}$ by $M(l)=\left\{f: A \rightarrow X \in\right.$ Ex mono $\left.\mathfrak{I} \mid A=l_{X} A\right\} . \quad M(l)$ satisfies the conditions $(M 2),(M 3)$ and $(A)$. However $M(l)$ must not satisfy the condition (M1) for a limit operator $l$ associated with a bicoreflective subcategory which is not closed under closed embeddings, e.g., the subcategory of all disjoint topological unions of connected spaces.

ExAMPLES. Let $M$ be a class of morphisms in $\mathfrak{T}$ such that $f \in M$ if and only if $f \in$ Iso $\mathfrak{I}$ or $f: \phi \rightarrow X$ for $X \in \mathrm{Ob} \mathfrak{T}$. Then $\mathfrak{I}\left(L_{\delta} M, M\right)$ is the bireflective subcategory of $\mathfrak{I}$ consisting of all indiscrete spaces.

(2) Let $(E, M)=\left(E_{0}, M_{0}\right)$. Then $\mathfrak{I}(E, M)=\mathfrak{I}$.

(3) Let $(E, M)=($ Epi $\mathfrak{T}$, Ex mono $\mathfrak{T})$. Then $\mathfrak{T}(E, M)$ is the bicoreffective subcategory of $\mathfrak{T}$ consisting of all discrete spaces.

(4) Let $M$ be a class of extremal monomorphisms in $\mathfrak{T}$ such that $f: A \rightarrow X \in M$ if and only if for any compact subspace $K$ of $X A \cap K$ is closed in $K$. Then we can show directly that $\left(L_{\delta} M, M\right)$ is an additive, strong and stable factorization structure on $\mathfrak{T} . \mathfrak{I}\left(L_{\delta} M, M\right)$ is the bicoreflective subcategory of $\mathfrak{I}$ consisting of all compactly generated spaces. The property 'compact' can be replaced by any map-invariant and closed-hereditary property, e.g., we have the category of sequential spaces and that of finitely generated spaces.

(5) Let $M^{\prime}$ be a class of all closed and open embedding in $\mathfrak{T}, E=L_{\delta} M^{\prime}$ and $M=R_{\delta} E$. Then $h: U \rightarrow V$ belongs to $E$ iff there exists no closed, open and proper subspace of $V$ containing $h(U)$. Let $f: X \rightarrow Y$ be a bimorphism satisfying the condition $\left(m_{0}\right)$ in Proposition 10 and let $j_{0}:: B \rightarrow B_{1}, j_{1}: B_{1} \rightarrow Y$ form the $(E, M)$-factorization of an extremal monomorphism $j: B \rightarrow Y$. Then the inverse images $i_{0}: f^{-1}(B) \rightarrow f^{-1}\left(B_{1}\right), i_{1}: f^{-1}\left(B_{1}\right) \rightarrow Y$ form the $(E, M)$-factorization of the inverse image $i: f^{-1}(B) \rightarrow Y$. Thus we have that $(E, M)$ is an additive, weak and stable factorization structure on $\mathfrak{T}$. $\mathfrak{I}(E, M)$ contains the bicoreflective subcategory of all disjoint topological unions of indiscrete spaces. The reals with usual topology is not contained in $\mathfrak{T}(E, M)$, but the subspace

is contained in $\mathfrak{T}(E, M)$.

$$
\{0\} \cup\left\{\frac{1}{n} \mid n=1,2, \cdots\right\}
$$


Let $X$ be a $T_{-1}$-space with a closure operation $t$. For a subset $A$ of $X$, let $\alpha=\left\{A_{\alpha_{i}}, i=1,2, \cdots, n_{\alpha}\right\}$ be a finite covering of $A$ and let $F_{A}$ be a set of all finite coverings of $A$. Define an operation $s$ by $s A=\bigcap_{\alpha \in F_{A}} \bigcup_{i=1}^{n_{m}} t A_{\alpha_{i}}$. Then $s$ gives a $T$-topology on the set $X$. The identity map on the set $X$ induces a continuous map $i_{X}:(X, s) \rightarrow(X, t)$ in $\mathfrak{I}_{-1}$. $\mathfrak{I}$ is a bicoreflective subcategory of $\mathfrak{I}_{-1}$ and $i_{X}$ is a $\mathfrak{I}$-coreflection of $X$. Let $(E, M)$ be the smallest additive factorization structure on $\mathfrak{I}_{-1}$ such that $(E, M)>\left(E_{0}, M_{0}\right)$, where $E_{0}$ and $M_{0}$ are classes of all dense maps and all closed embeddings in $\mathfrak{T}_{-1}$, respectively. Then an extremal monomorphism $f: A \rightarrow X$ belongs to $M$ if and only if $A=s A$. Hence we have that $(E, M)$ is stable and that $\mathfrak{I}_{-1}(E, M)=\mathfrak{I}$.

\section{References}

H. Herrlich (1968), Topologische Reflexionen und Coreflexionen, Lecture Note 78 (Springer, 1968).

H. Herrlich (1969), 'Limit operators and topological coreflections', Trans. Amer. Math. Soc. 146, 203-210.

H. Herrlich (1972), 'Coreflective subcategories in general topology', Fund. Math. 73, 199-218.

H. Herrlich (to appear). 'Perfect subcategories and factorizations'.

J. F. Kennison (1968), 'Full reflective subcategories and generalized covering spaces', Illinois J. Math. 12, 353-365.

B. Mitchell (1965), Theory of categories (Academic Press, New York, 1965).

G. E. Strecker (1972), 'Epireflection operator vs perfect morphisms and closed classes of epimorphisms', Bull. Austral. Math: Soc. 7, 359-366.

Department of Mathematics

Tokyo University of Education

Otsuka, Bunkyo-Ku, Tokyo. 